\title{
Searching for doubly charged Higgs bosons at the LHC in a 3-3-1 model
}

\author{
J.E. Cieza Montalvo a, Nelson V. Cortez Jr. ${ }^{\text {b,* }}$, J. Sá Borges a , \\ Mauro D. Tonasse ${ }^{\mathrm{c}}$ \\ a Instituto de Física, Universidade do Estado do Rio de Janeiro, \\ Rua São Francisco Xavier 524, 20559-900 Rio de Janeiro, RJ, Brazil \\ ${ }^{\mathrm{b}}$ Rua Justino Boschetti 40, 02205-050 São Paulo, SP, Brazil \\ c Campus Experimental de Registro, Universidade Estadual Paulista, \\ Rua Tamekishi Takano 5, 11900-000 Registro, SP, Brazil
}

Received 24 April 2006; received in revised form 12 August 2006; accepted 18 August 2006

Available online 7 September 2006

\begin{abstract}
Using a peculiar version of the $\mathrm{SU}(3)_{L} \otimes \mathrm{U}(1)_{N}$ electroweak model, we investigate the production of doubly charged Higgs boson at the Large Hadron Collider. Our results include branching ratio calculations for the doubly charged Higgs and for one of the neutral scalar bosons of the model.

(c) 2006 Elsevier B.V. All rights reserved.

PACS: 11.15.Ex; 12.60.Fr; 14.80.Cp
\end{abstract}

\section{Introduction}

In the last few years we have seen a tremendous experimental progress in the realm of the weak interactions. However, these advances do not attain the scalar sector yet. This is the sense in which LHC (Large Hadron Collider) facilities may shed some light especially on the Higgs boson. One of the main ingredients of the Standard Model is the Higgs mechanism which, in principle, explains how the particles gain masses through the introduction of an isodoublet scalar field. The scalar field is the responsible for the spontaneous breakdown of the gauge symmetry. After electroweak symmetry breaking, the interaction of this scalar with the gauge bosons and

\footnotetext{
* Corresponding author.

E-mail address: nelson.v.cortez@gmail.com (N.V. Cortez Jr.).
} 
fermions generate the mass of the particles. In this process there remains a single neutral scalar, manifesting itself as the Higgs particle.

The Standard Model possibly is a low energy effective theory which must be generalized by some GUT (grand unified theory). However, there are several motivations to extend the electroweak theory below the GUT scale. Supersymmetric models, for example, provide a solution to the hierarchy problem through the cancellation of the quadratic divergences via fermionic and bosonic loop contributions [1]. The little Higgs model, recently proposed, predicts that the Higgs boson is a pseudo-Goldstone boson of some approximate broken global symmetry [2]. Therefore, this model is also able to solve the naturalness problem of the Higgs mass. One of the main motivations for the left-right models and for the study of their phenomenological consequences is that in which the Higgs triplet representation furnishes a seesaw type neutrino mass matrix associated with the presence of a doubly charged Higgs boson [3]. Therefore, these models suggest a route to understanding the pattern of the neutrino masses.

Doubly charged Higgs bosons appear in several popular extensions of the Standard Model such as left-right symmetric models [3] and Higgs triplet models [4]. However, there is another interesting class of electroweak models which also predict particles like that. This class of models is called 3-3-1 models [5,6]. This is the simplest chiral extension of the Standard Model. It is able to solve the fermion family's replication problem through of a simple relation between the number of colors and the anomaly cancellation mechanism. It is important to notice that a solution to this problem is not furnished even in the context of the GUTs. The 3-3-1 models have other interesting features, as for example the upper bound on the Weinberg mixing angle, through the relation $\sin ^{2} \theta_{W}<1 / 4$. This feature does not happen in any kind of others electroweak models except GUTs, where the value of $\sin ^{2} \theta_{W}$ is predicted. This result leads to an upper bound for the energy scale of the model when this parameter is evolved to high values [7]. In a similar fashion as occurs in left-right model, the seesaw mechanism can be naturally incorporated in some versions of the 3-3-1 models [8].

No Higgs bosons have yet been found. In the meantime, it is the last brick that is lacking to finish the construction of the building of the standard electroweak theory. Besides, it is possible that the Higgs sector brings to light a non-standard physics.

Since that the 3-3-1 models are good candidates for physics beyond the Standard Model, it is interesting to evaluate if the future accelerators will produce events in sufficient numbers to detect some of the 3-3-1 Higgs bosons. In particular, there is an increasing interest in the phenomenology associated with doubly charged Higgs bosons, a kind that appears in models that admit scalars in triplet representation of the gauge group [9]. Here we are interested in one of such version of the 3-3-1 models for which the scalar fields come only in triplet representation [6,8]. It predicts three new neutrals, four single charged and two doubly charged Higgs bosons. These scalars can be disclosed in relatively low energies, which make them interesting for searches in the next generation of particle accelerators.

Our work is organized as follows. In Section 2 we summarize the relevant features of the model, in Section 3 we present the cross section calculations and in Section 4 we give our conclusions.

\section{Overview of the model}

The underlying electroweak symmetry group is $\mathrm{SU}(3)_{L} \otimes \mathrm{U}(1)_{N}$, where $N$ is the quantum number of the U(1) group. Therefore, the left-handed lepton matter content is $\left(v_{a}^{\prime} \ell_{a}^{\prime} \mathrm{L}_{a}^{\prime}\right)_{L}^{\mathrm{T}}$ transforming as $(\mathbf{3}, 0)$, where $a=e, \mu, \tau$ is a family index (we are using primes for the interaction 
eigenstates). $\mathrm{L}_{a L}^{\prime}$ are lepton fields which can be the charge conjugates $\ell_{a R}^{\prime}{ }^{C}$ [5], the antineutrinos $v_{L a}^{\prime}{ }^{C}[10]$ or heavy leptons $P_{a L}^{\prime+}\left(P_{a L}^{\prime+}=E_{L}^{\prime+}, M_{L}^{\prime+}, T_{L}^{\prime+}\right)[6]$.

The model of Ref. [6] has the simplest scalar sector for 3-3-1 models. In this version the charge operator is given by

$$
\frac{Q}{e}=\frac{1}{2}\left(\lambda_{3}-\sqrt{3} \lambda_{8}\right)+N
$$

where $\lambda_{3}$ and $\lambda_{8}$ are the diagonal Gell-Mann matrices and $e$ is the elementary electric charge. The right-handed charged leptons are introduced in singlet representation of $\mathrm{SU}(3)_{L}$ as $\ell_{a R}^{\prime-} \sim(\mathbf{1},-1)$ and $P_{a R}^{\prime+} \sim(\mathbf{1}, 1)$.

The quark sector is given by

$$
Q_{1 L}=\left(\begin{array}{c}
u_{1}^{\prime} \\
d_{1}^{\prime} \\
J_{1}
\end{array}\right)_{L} \sim\left(3, \frac{2}{3}\right), \quad Q_{\alpha L}=\left(\begin{array}{c}
d_{\alpha}^{\prime} \\
u_{\alpha}^{\prime} \\
J_{\alpha}^{\prime}
\end{array}\right)_{L} \sim\left(3^{*},-\frac{1}{3}\right),
$$

where $\alpha=2,3, J_{1}$ and $J_{\alpha}$ are exotic quarks with electric charge $\frac{5}{3}$ and $-\frac{4}{3}$ respectively. It must be notice that the first quark family transforms differently from the two others under the gauge group, which is essential for the anomaly cancellation mechanism [5].

The physical fermionic eigenstates rise by the transformations

$$
\begin{aligned}
& \ell_{a L, R}^{\prime-}=A_{a b}^{L, R} \ell_{b L, R}^{-}, \quad P_{a L, R}^{++}=B_{a b}^{L, R} P_{b L, R}^{+}, \\
& U_{L, R}^{\prime}=\mathcal{U}^{L, R} U_{L, R}, \quad D_{L, R}^{\prime}=\mathcal{D}^{L, R} D_{L, R}, \quad J_{L, R}^{\prime}=\mathcal{J}^{L, R} J_{L, R},
\end{aligned}
$$

where $U_{L, R}=\left(\begin{array}{lll}u & c & t\end{array}\right)_{L, R}, D_{L, R}=\left(\begin{array}{lll}d & s & b\end{array}\right)_{L, R}, J_{L, R}=\left(\begin{array}{lll}J_{1} & J_{2} & J_{3}\end{array}\right)_{L, R}$ and $A^{L, R}, B^{L, R}, \mathcal{U}^{L, R}$, $\mathcal{D}^{L, R}, \mathcal{J}^{L, R}$ are arbitrary mixing matrices.

The minimal scalar sector contains the three scalar triplets

$$
\eta=\left(\begin{array}{c}
\eta^{0} \\
\eta_{1}^{-} \\
\eta_{2}^{+}
\end{array}\right) \sim(\mathbf{3}, 0), \quad \rho=\left(\begin{array}{c}
\rho^{+} \\
\rho^{0} \\
\rho^{++}
\end{array}\right) \sim(3,1), \quad \chi=\left(\begin{array}{c}
\chi^{-} \\
\chi^{--} \\
\chi^{0}
\end{array}\right) \sim(3,-1) .
$$

The most general, gauge invariant and renormalizable Higgs potential, which conserves the leptobaryon number [11], is

$$
\begin{aligned}
V(\eta, \rho, \chi)= & \mu_{1}^{2} \eta^{\dagger} \eta+\mu_{2}^{2} \rho^{\dagger} \rho+\mu_{3}^{2} \chi^{\dagger} \chi+\lambda_{1}\left(\eta^{\dagger} \eta\right)^{2}+\lambda_{2}\left(\rho^{\dagger} \rho\right)^{2}+\lambda_{3}\left(\chi^{\dagger} \chi\right)^{2} \\
& +\left(\eta^{\dagger} \eta\right)\left[\lambda_{4}\left(\rho^{\dagger} \rho\right)+\lambda_{5}\left(\chi^{\dagger} \chi\right)\right]+\lambda_{6}\left(\rho^{\dagger} \rho\right)\left(\chi^{\dagger} \chi\right)+\lambda_{7}\left(\rho^{\dagger} \eta\right)\left(\eta^{\dagger} \rho\right) \\
& +\lambda_{8}\left(\chi^{\dagger} \eta\right)\left(\eta^{\dagger} \chi\right)+\lambda_{9}\left(\rho^{\dagger} \chi\right)\left(\chi^{\dagger} \rho\right)+\frac{1}{2}\left(f \epsilon^{i j k} \eta_{i} \rho_{j} \chi_{k}+\text { H.c. }\right) .
\end{aligned}
$$

The neutral components of the scalars triplets (4) develop non-zero vacuum spectration values $\left\langle\eta^{0}\right\rangle=v_{\eta},\left\langle\rho^{0}\right\rangle=v_{\rho}$ and $\left\langle\chi^{0}\right\rangle=v_{\chi}$, with $v_{\eta}^{2}+v_{\rho}^{2}=v_{W}^{2}=(246 \mathrm{GeV})^{2}$. This mechanism generate the fermion and gauge boson masses [12]. The pattern of symmetry breaking is $\mathrm{SU}(3)_{L} \otimes \mathrm{U}(1)_{N} \stackrel{\langle\chi\rangle}{\longmapsto} \mathrm{SU}(2)_{L} \otimes \mathrm{U}(1)_{Y} \stackrel{\langle\eta, \rho\rangle}{\longmapsto} \mathrm{U}(1)_{\mathrm{em}}$. Therefore, we can expect $v_{\chi} \gg v_{\eta}, v_{\rho}$. In the potential (5) $f$ and $\mu_{j}(j=1,2,3)$ are constants with dimension of mass and the $\lambda_{i}$ $(i=1, \ldots, 9)$ are adimensional constants with $\lambda_{3}<0$ and $f<0$ from the positivity of the scalar masses [12]. The $\eta$ and $\rho$ scalar triplets give masses to the ordinary fermions and gauge bosons, while the $\chi$ scalar triplet gives masses to the new fermion and gauge bosons. In this work we are 
using the eigenstates and masses (see Appendix B) of Ref. [12]. For others analysis of the 3-3-1 Higgs potential see Ref. [13].

Symmetry breaking is initiated when the scalar neutral fields are shifted as $\varphi=v_{\varphi}+\xi_{\varphi}+i \zeta_{\varphi}$, with $\varphi=\eta^{0}, \rho^{0}, \chi^{0}$. Thus, the physical neutral scalar eigenstates $H_{1}^{0}, H_{2}^{0}, H_{3}^{0}$ and $h^{0}$ are related to the shifted fields as

$$
\left(\begin{array}{l}
\xi_{\eta} \\
\xi_{\rho}
\end{array}\right) \approx \frac{1}{v_{W}}\left(\begin{array}{cc}
c_{\omega} & s_{\omega} \\
s_{\omega} & -c_{\omega}
\end{array}\right)\left(\begin{array}{c}
H_{1}^{0} \\
H_{2}^{0}
\end{array}\right), \quad \xi_{\chi} \approx H_{3}^{0}, \quad \zeta_{\chi} \approx i h^{0},
$$

and in the charged scalar sector we have

$$
\begin{array}{ll}
\eta_{1}^{+}=s_{\omega} H_{1}^{+}, & \eta_{2}^{+}=s_{\varphi} H_{2}^{+}, \quad \rho^{+}=c_{\omega} H_{1}^{+}, \\
\chi^{+}=c_{\varphi} H_{2}^{+}, & \rho^{++}=s_{\phi} H^{++}, \quad \chi^{++}=c_{\phi} H^{++},
\end{array}
$$

with the condition that $v_{\chi} \gg v_{\eta}, v_{\rho}$ in Eqs. (6a) and $c_{\omega}=\cos \omega=v_{\eta} / \sqrt{v_{\eta}^{2}+v_{\rho}^{2}}, s_{\omega}=\sin \omega$, $c_{\phi}=\cos \phi=v_{\rho} / \sqrt{v_{\rho}^{2}+v_{\chi}^{2}}, s_{\phi}=\sin \phi, c_{\varphi}=\cos \varphi=v_{\eta} / \sqrt{v_{\eta}^{2}+v_{\chi}^{2}}, s_{\varphi}=\sin \varphi$. The $H_{1}^{0}$ Higgs boson in Eq. (6a) can be the Standard Model scalar boson, since its mass (see Appendix B) has no dependence on the spectration value $v_{\chi}[12]$.

The Yukawa interactions for leptons and quarks are, respectively,

$$
\begin{aligned}
-\mathcal{L}_{\ell}= & G_{a b} \bar{\psi}_{a L} \ell_{b R}^{\prime} \rho+G_{a b}^{\prime} \bar{\psi}_{a L} P_{b R}^{\prime} \chi+\text { H.c. } \\
-\mathcal{L}_{Q}= & \bar{Q}_{1 L} \sum_{i}\left[G_{1 i}^{u} U_{i R}^{\prime} \eta+G_{1 i}^{d} D_{i R}^{\prime} \rho+\sum_{\alpha} \bar{Q}_{\alpha L}\left(F_{\alpha i}^{u} U_{i R}^{\prime} \rho^{*}+F_{\alpha i}^{d} D_{i R}^{\prime} \eta^{*}\right)\right] \\
& +G^{j} \bar{Q}_{1 L} J_{1 R} \chi+\sum_{\alpha \beta} G_{\alpha \beta}^{j} \bar{Q}_{\alpha L} J_{\beta R}^{\prime} \chi^{*}+\text { H.c. }
\end{aligned}
$$

In Eqs. (7), as before mentioned $a, b=e, \mu, \tau$ and $\alpha=2,3$.

Beyond the standard particles $\gamma, Z$ and $W^{ \pm}$the model predicts, in the gauge sector, one neutral $\left(Z^{\prime}\right)$, two single charged $\left(V^{ \pm}\right)$and two double charged $\left(U^{ \pm \pm}\right)$gauge bosons. The interactions between the gauge and Higgs bosons are given by the covariant derivative

$$
\mathcal{D}_{\mu} \varphi_{i}=\partial_{\mu} \varphi_{i}-i g\left(\vec{W}_{\mu} \cdot \frac{\vec{\lambda}}{2}\right)_{i}^{j} \varphi_{j}-i g^{\prime} N_{\varphi} \varphi_{i} B_{\mu},
$$

where $N_{\varphi}$ are the $\mathrm{U}(1)$ charges for the $\varphi$ Higgs triplets $(\varphi=\eta, \rho, \chi) . \vec{W}_{\mu}$ and $B_{\mu}$ are field tensors of SU(2) and U(1), respectively, $\vec{\lambda}$ are Gell-Mann matrices and $g$ and $g^{\prime}$ are coupling constants for $\mathrm{SU}(2)$ and $U(1)$, respectively.

Introducing the eigenstates (3) and (6c) in the Lagrangians (7b) we obtain the Yukawa interactions as function of the physical eigenstates, i.e.,

$$
\begin{aligned}
-\mathcal{L}_{\ell}= & \frac{1}{2}\left\{\frac{1}{v_{\rho}}\left[c_{\omega} \bar{\nu} \mathcal{U}^{\nu e} H_{1}^{+}+\left(v_{\rho}+s_{\omega} H_{1}^{0}-c_{\omega} H_{2}^{0}\right) \overline{e^{-}}+s_{\phi} \overline{P^{+}} \mathcal{U}^{P e} H^{++}\right] M^{e} G_{R} e^{-}\right. \\
& \left.+\frac{1}{v_{\chi}}\left[c_{\omega} \bar{\nu} \mathcal{V}^{\nu P} H_{2}^{-}+c_{\phi} \overline{e^{-}} \mathcal{V}^{e P} H^{--}+\left(v_{\chi}+H_{3}^{0}+i h^{0}\right) \overline{P^{+}}\right] M^{E} G_{R} P^{+}\right\} \\
& + \text {H.c. }
\end{aligned}
$$




$$
\begin{aligned}
-\mathcal{L}_{Q}= & \frac{1}{2}\left\{\bar{U} G_{R}\left[1+\left[\frac{s_{\omega}}{v_{\rho}}+\left(\frac{c_{\omega}}{v_{\eta}}+\frac{s_{\omega}}{v_{\rho}}\right) \mathcal{V}^{u}\right] H_{1}^{0}+\left[-\frac{c_{\omega}}{v_{\rho}}+\left(\frac{s_{\omega}}{v_{\eta}}-\frac{c_{\omega}}{v_{\rho}}\right) \mathcal{V}^{u}\right] H_{2}^{0}\right] M^{u} U\right. \\
& +\bar{D} G_{R}\left[1+\left[\frac{c_{\omega}}{v_{\eta}}+\left(\frac{s_{\omega}}{v_{\rho}}-\frac{c_{\omega}}{v_{\eta}}\right) \mathcal{V}^{D}\right] H_{1}^{0}+\left[\frac{s_{\omega}}{v_{\eta}}-\left(\frac{c_{\omega}}{v_{\rho}}+\frac{s_{\omega}}{v_{\eta}}\right) \mathcal{V}^{D}\right] H_{2}^{0}\right] M^{d} D \\
& +\bar{U} G_{R}\left[\frac{s_{\omega}}{v_{\eta}} V_{\mathrm{CKM}}^{\dagger} H_{1}^{-}+\left(\frac{c_{\omega}}{v_{\eta}}-\frac{s_{\omega}}{v_{\rho}}\right) \mathcal{V}^{u d} H_{1}^{+}\right] M^{d} D \\
& \left.+\bar{D} G_{R}\left[\frac{c_{\omega}}{v_{\rho}} V_{\mathrm{CKM}} H_{1}^{+}+\left(\frac{s_{\omega}}{v_{\eta}}-\frac{c_{\omega}}{v_{\rho}}\right) \mathcal{V}^{u d \dagger} H_{1}^{-}\right] M^{u} U\right\}+ \text { H.c., } \\
-\mathcal{L}_{J}= & \frac{1}{2}\left[\bar{J} G_{R} \mathcal{J}^{L \dagger}\left(\mathcal{N} \mathcal{U}^{L} M^{u} U+\mathcal{R} \mathcal{D}^{L} M^{d} D\right)\right. \\
& \left.+\left(\bar{U} \mathcal{U}^{L \dagger} \mathcal{X}_{1}+\bar{D} \mathcal{D}^{L \dagger} \mathcal{X}_{2}+\bar{J} \mathcal{J}^{L \dagger} \mathcal{X}_{0}\right) \mathcal{J}^{L} M^{J} G_{R} J\right]+ \text { H.c., }
\end{aligned}
$$

where $G_{R}=1+\gamma_{5}, V_{L}^{U} V_{L}^{D}=V_{\mathrm{CKM}}$, the Cabibbo-Kobayashi-Maskawa mixing matrix, $\mathcal{U}^{v e}$, $\mathcal{U}^{P e}, \mathcal{V}^{v e}, \mathcal{V}^{e P}, \mathcal{V}^{u}=V_{L}^{U} \Delta V_{L}^{U \dagger}, \mathcal{V}^{d}=V_{L}^{D} \Delta V_{L}^{D^{\dagger}}$ and $\mathcal{V}^{u d}=V_{L}^{U} \Delta V_{L}^{D \dagger}$ are arbitrary mixing matrices, $M^{e}=\operatorname{diag}\left(m_{e} m_{\mu} m_{\tau}\right), M^{P}=\operatorname{diag}\left(m_{E} m_{M} m_{T}\right), M^{u}=\operatorname{diag}\left(m_{u} m_{c} m_{t}\right), M^{d}=$ $\operatorname{diag}\left(m_{d} m_{s} m_{b}\right)$ and $M^{J}=\operatorname{diag}\left(m_{J_{1}} m_{J_{2}} m_{J_{3}}\right)$. In Eq. (9c) we have defined

$$
\begin{aligned}
\mathcal{N} & =\left(\begin{array}{ccc}
s_{\omega} H_{2}^{+} / v_{\eta} & 0 & 0 \\
0 & s_{\phi} H^{--} / v_{\rho} & s_{\phi} H^{--} / v_{\rho} \\
0 & s_{\phi} H^{--} / v_{\rho} & s_{\phi} H^{--} / v_{\rho}
\end{array}\right), \\
\mathcal{X}_{0} \approx & \frac{v_{\chi}+H_{3}^{0}+i h^{0}}{v_{\chi}}\left(\begin{array}{ccc}
1 & 0 & 0 \\
0 & 1 & 1 \\
0 & 1 & 1
\end{array}\right) \\
\mathcal{R} & =\left(\begin{array}{ccc}
s_{\phi} H^{++} / v_{\rho} & 0 & 0 \\
0 & s_{\omega} H_{2}^{-} / v_{\eta} & s_{\omega} H_{2}^{-} / v_{\eta} \\
0 & s_{\omega} H_{2}^{-} / v_{\eta} & s_{\omega} H_{2}^{-} / v_{\eta}
\end{array}\right), \\
\mathcal{X}_{1} & =\frac{1}{v_{\chi}}\left(\begin{array}{ccc}
c_{\omega} H_{2}^{-} & 0 & 0 \\
0 & c_{\phi} H^{++} & c_{\phi} H^{++} \\
0 & c_{\phi} H^{++} & c_{\phi} H^{++}
\end{array}\right) \\
\mathcal{X}_{2} & =\frac{1}{v_{\chi}}\left(\begin{array}{ccc}
c_{\phi} H^{--} & 0 & 0 \\
0 & c_{\omega} H_{2}^{+} & c_{\omega} H_{2}^{+} \\
0 & c_{\omega} H_{2}^{+} & c_{\omega} H_{2}^{+}
\end{array}\right) .
\end{aligned}
$$

We call attention to the fact that non-standard field interactions violate leptonic number, as can be seen from the Lagrangians (7b) and (8). However the total leptonic number is conserved [5].

\section{Cross section production}

The main mechanism for the production of Higgs particles in $p p$ collisions occurs through the mechanism of Drell-Yan and gluon-gluon fusion as shown in Fig. 1. In all calculations we are considering that the charged fermionic mixing matrices [see Eqs. (3)] are diagonals. Using the interaction Lagrangians (5) and (9) we first evaluate the differential cross section for the Drell-Yan process, i.e., $p p \rightarrow H^{++} H^{--}$through the exchange of $\gamma, \mathrm{Z}, Z^{\prime}, H_{1}^{0}$ and $H_{2}^{0}$ in the 


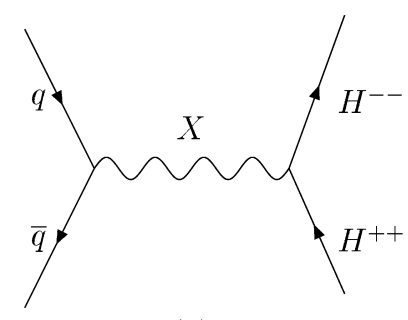

(a)

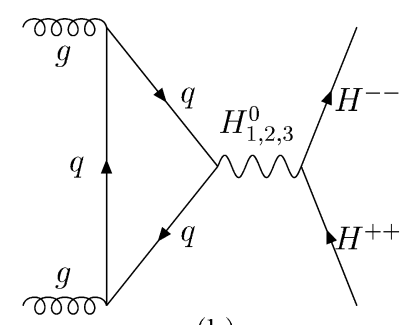

(b)

Fig. 1. Feynman diagrams for production of doubly charged Higgs bosons via (a) Drell-Yan process, where $X=\gamma, Z, Z^{\prime}, H_{1}^{0}, H_{2}^{0}$ and (b) gluon-gluon fusion.

$s$-channel. Therefore we obtain the differential cross section for this reaction as

$$
\frac{d \hat{\sigma}}{d \Omega}=\frac{1}{64 \pi^{2} \hat{s}}\left(\overline{\left|\mathcal{M}_{\gamma}\right|^{2}}+\overline{\left|\mathcal{M}_{H_{1}^{0}}\right|^{2}}+\overline{\left|\mathcal{M}_{H_{2}^{0}}\right|^{2}}+\overline{\left|\mathcal{M}_{Z}\right|^{2}}+\overline{\left|\mathcal{M}_{Z^{\prime}}\right|^{2}}+2 \operatorname{Re} \overline{\mathcal{M}_{H_{1}^{0}} \mathcal{M}_{H_{2}^{0}}{ }^{*}}\right),
$$

where

$$
\begin{aligned}
\frac{d \hat{\sigma}}{d \cos \theta}= & \frac{\beta_{H^{ \pm \pm}}}{24}\left\{\frac{\left[\Lambda_{1} \zeta^{(1)}(\hat{s}) m_{q} v_{\eta} v_{\rho}\right]^{2}+\left[\Lambda_{2} \zeta^{(2)}(\hat{s})\right]^{2}\left(v_{\eta}^{4} m_{u}^{2}+v_{\rho}^{4} m_{d}^{2}\right)}{\left(2 v_{W} v_{\eta} v_{\rho}\right)^{2}}\right. \\
& \times\left[8\left(m_{q}^{2}+m_{H^{ \pm \pm}}^{2}\right)-4 \hat{u}-4 \hat{t}\right] \\
& \left.+\frac{2 \pi}{\hat{s}^{3}}\left(\frac{\Lambda \alpha Q_{q}}{\sin \theta_{W}}\right)^{2}\left[\left(\hat{s}-2 m_{q}^{2}\right) \hat{s}-4 m_{H^{ \pm \pm}}^{2}\left(\hat{s}+2 m_{q}^{2}\right)-(\hat{t}-\hat{u})^{2}\right]\right\} \\
& +\sum_{Z, Z^{\prime}} \frac{\beta_{H^{ \pm \pm}} \alpha^{2} \pi \Lambda_{Z\left(Z^{\prime}\right)}^{2}}{36 \sin ^{2} \theta_{W} \cos ^{2} \theta_{W} \hat{s}\left(\hat{s}-m_{Z\left(Z^{\prime}\right)}^{2}+i m_{Z\left(Z^{\prime}\right)} \Gamma_{\left.Z\left(Z^{\prime}\right)\right)^{2}}\right.} \\
& \times\left[8 m_{H^{ \pm \pm}}^{4}\left(g_{V}^{q\left(q^{\prime}\right) 2}+g_{A}^{q\left(q^{\prime}\right) 2}\right)\right. \\
& +8 m_{H^{ \pm \pm}}^{2}\left(2 m_{q}^{2}-\hat{t}-\hat{u}\right)\left(g_{V}^{q\left(q^{\prime}\right) 2}+g_{A}^{q\left(q^{\prime}\right) 2}\right)+8 m_{q}^{4}\left(g_{V}^{q\left(q^{\prime}\right) 2}+g_{A}^{q\left(q^{\prime}\right) 2}\right) \\
& -8 m_{q}^{2}(\hat{t}+\hat{u})\left(g_{V}^{q\left(q^{\prime}\right) 2}+g_{A}^{q\left(q^{\prime}\right) 2}\right)+8 m_{q}^{2} \hat{s} g_{A}^{q\left(q^{\prime}\right) 2} \\
& \left.+2(\hat{t}+\hat{u})^{2}\left(g_{V}^{q\left(q^{\prime}\right) 2}+g_{A}^{q\left(q^{\prime}\right) 2}\right)+-2 \hat{s}^{2}\left(g_{V}^{q\left(q^{\prime}\right) 2}+g_{A}^{q\left(q^{\prime}\right) 2}\right)\right] .
\end{aligned}
$$

Here, $\sqrt{\hat{s}}$ is the CM (center of mass) energy of the $q \bar{q}$ system. For the Standard Model parameters we assume PDG values, i.e., $m_{Z}=91.19 \mathrm{GeV}, \sin ^{2} \theta_{W}=0.2315$, and $m_{W}=80.33 \mathrm{GeV}$ [14]. $\Gamma_{Z\left(Z^{\prime}\right)}$ are the total width of the boson $Z\left(Z^{\prime}\right)[15,16]$. The velocity of the Higgs in the CM of the process is denoted through $\beta_{H^{ \pm \pm}}$. The $\Lambda_{i}(i=1,2)$ are the vertex strengths for $H_{1}^{0} H^{--} H^{++}$ and $H_{2}^{0} H^{--} H^{++}$, respectively, while $\Lambda_{\gamma_{\mu}}$ is one for $\gamma H^{--} H^{++}$and the $\Lambda_{Z\left(Z^{\prime}\right)_{\mu}}$ is for the bosons $Z\left(Z^{\prime}\right) H^{--} H^{++}$. The analytical expressions for these vertex strengths are

$$
\begin{aligned}
\Lambda_{1}= & -2 i\left\{2\left[\left(\lambda_{6}+\lambda_{9}\right) c_{\phi}^{2}+\left(2 \lambda_{2}+\lambda_{9}\right) s_{\phi}^{2}\right] s_{\omega} v_{\rho}\right. \\
& \left.+c_{\omega}\left[2\left(\lambda_{5} c_{\phi}^{2}+\lambda_{4} s_{\phi}^{2}\right) v_{\eta}+-f c_{\phi} s_{\phi}\right]\right\}, \\
\Lambda_{2}= & i c_{\omega}\left\{2\left[\left(-\lambda_{5}+\lambda_{6}+\lambda_{9}\right) c_{\phi}^{2}+2\left(2 \lambda_{2}-\lambda_{4}-\lambda_{9}\right) s_{\omega}\right] v_{\eta}+f c_{\phi} s_{\phi}\right\}, \\
\Lambda_{\gamma \mu}= & -e\left(c_{\phi}^{2}-s_{\phi}^{2}\right)(p-q)_{\mu},
\end{aligned}
$$




$$
\begin{aligned}
& \Lambda_{Z \mu}=-e \frac{4 \sin ^{2} \theta_{W}\left(v_{\eta}^{2}-v_{\chi}^{2}\right)-v_{\eta}^{2}}{4\left(v_{\eta}^{2}+v_{\chi}^{2}\right) \sin \theta_{W} \cos \theta_{W}}(p-q)_{\mu}, \\
& \Lambda_{Z^{\prime} \mu}=-e \frac{\left(10 \sin ^{2} \theta_{W}-1\right) v_{\eta}^{2}+\left(1-7 \sin ^{2} \theta_{W}\right) v_{\chi}^{2}}{4\left(v_{\eta}^{2}+v_{\chi}^{2}\right) \sin \theta_{W} \sqrt{3 \cos ^{2} \theta_{W}\left(1-4 \sin ^{2} \theta_{W}\right)}}(p-q)_{\mu} .
\end{aligned}
$$

The Higgs parameters $\lambda_{i}(i=1, \ldots, 9)$ must run from -3 to +3 in order to allow perturbative calculations. For $H_{\alpha}^{0}(\alpha=2,3)$ we take $m_{H_{\alpha}^{0}}=(0.2-3.0) \mathrm{TeV}$, while we assume $m_{H_{1}^{0}}=230 \mathrm{GeV}$ for the Standard Model scalar one. It must be notice that here there is no contribution from the interference between the scalar particle $H_{1}^{0}$ and a vectorial one $\left(\gamma, Z\right.$ or $\left.Z^{\prime}\right)$. The kinematic invariant $\hat{t}$ and $\hat{u}$ are,

$$
\begin{aligned}
& \hat{t}=m_{q}^{2}+m_{H_{ \pm \pm}}^{2}-\frac{\hat{s}}{2}\left(1-\cos \theta \sqrt{1-\frac{4 m_{q}^{2}}{\hat{s}}} \sqrt{1-\frac{4 m_{H^{ \pm \pm}}^{2}}{\hat{s}}}\right), \\
& \hat{u}=m_{q}^{2}+m_{H_{ \pm \pm}}^{2}-\frac{\hat{s}}{2}\left(1+\cos \theta \sqrt{1-\frac{4 m_{q}^{2}}{\hat{s}}} \sqrt{1-\frac{4 m_{H^{ \pm \pm}}^{2}}{\hat{s}}}\right) .
\end{aligned}
$$

The total cross section $(\sigma)$ for the process $p p \rightarrow H^{++} H^{--}$is related to the cross section $(\hat{\sigma})$ of the subprocess $q q \rightarrow H^{++} H^{--}$through

$$
\sigma=\sum_{q_{i}=u, d, s, c} \int_{\tau_{\min }}^{1} \int_{\ln \sqrt{\tau_{\min }}}^{-\ln \sqrt{\tau_{\min }}} d \tau d y f_{q_{i}}\left(\sqrt{\tau} e^{y}\right) f_{\bar{q}_{i}}\left(\sqrt{\tau} e^{-y}\right) \hat{\sigma}(\tau, \hat{s}),
$$

where $\tau_{\min }=\hat{s} / s$ and $f_{q i}$ and $f_{\bar{q} i}$ are the structure functions of the quark and antiquark in the proton, for which the factorization scale is taken equal to the center of mass energy of the $q \bar{q}$ system and used in our numerical calculations.

Another form to produce a pair of doubly charged Higgs is via the gluon-gluon fusion through the reaction $g g \rightarrow H^{++} H^{--}$(see Fig. 1(b)). As the final state is neutral, the $s$-channel involves the exchange of the three neutral Higgs $H_{1}^{0}, H_{2}^{0}$ and $H_{3}^{0}$. The exchange of a photon is not allowed by $C$ conservation (Furry's theorem). Therefore, the differential cross section for gluon-gluon fusion can be expressed by

$$
\begin{aligned}
\frac{d \hat{\sigma}}{d \Omega}= & \frac{1}{64 \pi^{2} \hat{s}}\left[\overline{\left|\mathcal{M}_{H_{1}^{0}}\right|^{2}}+\overline{\left|\mathcal{M}_{H_{2}^{0}}\right|^{2}}+\overline{\left|\mathcal{M}_{H_{3}^{0}}\right|^{2}}\right. \\
& \left.+2\left(\operatorname{Re} \overline{\mathcal{M}_{H_{1}^{0}} \mathcal{M}_{H_{2}^{0}}^{*}}+\operatorname{Re} \overline{\mathcal{M}_{H_{1}^{0}} \mathcal{M}_{H_{3}^{*}}^{*}}+\operatorname{Re} \overline{\mathcal{M}_{H_{2}^{0}} \mathcal{M}_{H_{3}^{0}}^{*}}\right)\right] .
\end{aligned}
$$

In order to make explicit the different contributions to the elementary cross section, we will present them separately. The quark-loop contributions involve the Higgs $H_{i}$, where $i=1,2,3$, which are exchanged in the $s$-channel. For the $H_{1}^{0}$ we have

$$
\begin{aligned}
\hat{\sigma}_{H_{1}^{0}}= & \frac{\beta_{H^{++}}}{8192 \pi^{3}}\left[\alpha_{s} \Lambda_{1} \zeta^{(1)}\right]^{2}\left\{\frac{1}{\hat{s}}\left|\sum_{q} \Lambda_{q_{1}} m_{q}^{2} I_{d}\right|^{2}+\hat{s}\left|\sum_{q} \Lambda_{q_{1}} \lambda_{q}\left[2+\left(4 \lambda_{q}-1\right) I_{q}\right]\right|^{2}\right. \\
& \left.-2 \pi \sum_{q} m_{q}^{2} \lambda_{q} \ln \left(\frac{r_{+q}}{r_{-q}}\right)\left[\ln ^{2}\left(\frac{r_{+q}}{r_{-q}}\right)-\pi^{2}\right]\right\} .
\end{aligned}
$$


We fix the scale parameter $\Lambda=0.2 \mathrm{GeV}$ and the appropriate scale where the strong coupling constant $\alpha_{s}$ is evaluated as being equal to the center of mass energy of the subprocess, both, used in our numerical calculations. The sums in Eq. (16) run over all generations where $\lambda_{q}=m_{q}^{2} / \hat{s}$ and $r_{ \pm q}=1 \pm \sqrt{1-4 \lambda_{q}}$. The $\Lambda_{q_{1}}$ is the $q \bar{q} H_{1}^{0}$ vertex strength,

$$
\Lambda_{q_{1}}=-i \frac{m_{q}}{2 v_{W}} G_{R}
$$

We also define the propagator for Higgs bosons,

$$
\zeta^{(i)}(\hat{s})=\frac{1}{\hat{s}-m_{H_{i}}^{2}+i m_{H_{i}} \Gamma_{H_{i}}},
$$

where $\Gamma_{H_{i}^{0}}$ are the total width of the $H_{1}^{0}$ and $H_{2}^{0}$ boson, with $i=1$ for $q=u, c, t, d, s, b$ and $i=2$ for $u(c, t)$ or $d(s, b)$, separately [16]. Eq. (18) defines also the propagator for $H_{3}^{0}$ with $i=3$. However, $H_{3}^{0}$ does not contribute to the Drell-Yan process.

For the Higgs $H_{2}^{0}$ we have

$$
\begin{aligned}
\hat{\sigma}_{H_{2}^{0}}= & \frac{\beta_{H^{++}}}{8192 \pi^{3}}\left[\alpha_{s} \Lambda_{2}\left|\zeta^{(2)}(\hat{s})\right|\right]^{2}\left\{\frac{1}{\hat{s}} \sum_{q} \Lambda_{q_{2}}\left(m_{u}^{2} \frac{v_{\eta}}{v_{\rho}}+m_{d}^{2} \frac{v_{\rho}}{v_{\eta}}\right)^{2}\left|I_{q}\right|^{2}\right. \\
& +\hat{s}\left(\frac{v_{\eta}}{v_{\rho}}+\frac{v_{\rho}}{v_{\eta}}\right)^{2}\left|\sum_{q} \Lambda_{q_{2}} \lambda_{q}\left[2+\left(4 \lambda_{q}-1\right) I_{q}\right]\right|^{2} \\
& \left.+4 \pi \sum_{q} \Lambda_{q_{2}} \ln \left(\frac{r_{+q}}{r_{-q}}\right)\left(-m_{u}^{2} \frac{v_{\eta}^{2}}{v_{\rho}^{2}}+m_{d}^{2} \frac{v_{\rho}^{2}}{v_{\eta}^{2}}\right) \lambda_{q}\right\},
\end{aligned}
$$

where $\Lambda_{q_{2}}$ is the strength of the $q \bar{q} H_{2}^{0}$ vertex, i.e.:

$$
\Lambda_{q_{2}}=-\frac{i}{2 v_{W}} G_{R}\left(m_{u} \frac{v_{\eta}}{v_{\rho}}+m_{d} \frac{v_{\rho}}{v_{\eta}}\right) .
$$

The contribution of $H_{3}^{0}$ to the cross section is

$$
\hat{\sigma}_{H_{3}^{0}}=\frac{\beta^{H^{++}}}{8192 \pi^{3}}\left[\alpha_{2} \Lambda_{3}\left|\zeta^{(3)}\right|\right]^{2}\left|\sum_{J=J_{1}, J_{2}, J_{3}} \Lambda_{J 3} \lambda_{J}\left[2+\left(4 \lambda_{J}-1\right) I_{J}\right]\right|^{2},
$$

where $\Lambda_{J 3}$ describe the quark vertex with the Higgs $H_{3}^{0}$ and $\Lambda_{3}$ for the $H_{3}^{0}$ with $H^{ \pm \pm}$, i.e.,

$$
\begin{aligned}
& \Lambda_{J 3}=-i \frac{m_{J}}{2 v_{\chi}} G_{R}, \\
& \Lambda_{3}=-2 i v_{\chi}\left[\left(\lambda_{6}-\lambda_{9}\right) s_{\varphi}^{2}+\left(2 \lambda_{3}+\lambda_{9}\right) c_{\phi}^{2}\right] .
\end{aligned}
$$

We note that $H_{3}^{0}$ decays only into exotic leptons and quarks because it becomes massive at the first symmetry breaking. Therefore, the $H_{3}^{0}$ total width is obtained from

$$
\begin{aligned}
\Gamma\left(H_{3}^{0} \rightarrow \text { all }\right)= & \Gamma_{J_{1} \bar{J}_{1}}^{0}+\Gamma_{J_{2} \bar{J}_{2}}^{0}+\Gamma_{J_{3} \bar{J}_{3}}^{0}+3 \Gamma_{P^{ \pm} P^{\mp}}^{0}+\Gamma_{H_{1}^{0} H_{2}^{0}}^{0}+\Gamma_{H_{1}^{ \pm} H_{1}^{\mp}}^{0} \\
& +\Gamma_{H_{2}^{ \pm} H_{2}^{\mp}}^{0}+\Gamma_{H_{1}^{ \pm} H_{2}^{\mp}}^{0}+\Gamma_{U^{\mp \mp} U^{ \pm \pm}}^{0}
\end{aligned}
$$


where $\Gamma_{X Y}^{0}=\Gamma\left(H_{3}^{0} \rightarrow X Y\right)$. The partial widths are shown in Appendix A.

The total width of the decay of the Higgs $H^{ \pm \pm}$in quarks, leptons, standard charged gauge boson and charged Higgs $\left(W^{ \pm} H_{2}^{ \pm}\right)$, single charged Higgs $\left(H_{1}^{ \pm} H_{2}^{ \pm}\right)$, doubly charged gauge bosons and a photon $\left(U^{ \pm \pm} \gamma\right)$, doubly charged bosons and Higgs $\left(U^{ \pm \pm} h^{0}, U^{ \pm \pm} H_{1}^{0}, U^{ \pm \pm} H_{2}^{0}, U^{ \pm \pm} H_{3}^{0}\right)$, doubly charged bosons and $Z$ or $Z^{\prime}\left(U^{ \pm \pm} Z, U^{ \pm \pm} Z^{\prime}\right)$, and charged extra gauge boson and Higgs $\left(V^{ \pm} H_{1}^{ \pm}\right)$is given by

$$
\begin{aligned}
\Gamma\left(H^{ \pm \pm} \rightarrow \text { all }\right)= & \Gamma_{\bar{J}_{1} q_{d, s, b}^{ \pm \pm}}^{ \pm}+\Gamma_{\bar{q}_{u, c, t} J_{2,3}}^{ \pm \pm}+\Gamma_{\bar{J}_{2,3} q_{u, c, t}}^{ \pm \pm}+\Gamma_{e^{ \pm} P^{ \pm \pm}}^{ \pm \pm}+\Gamma_{W^{ \pm} H_{2}^{ \pm}}^{ \pm \pm}+\Gamma_{H_{1}^{ \pm}}^{ \pm \pm} H_{2}^{ \pm} \\
& +\Gamma_{U^{ \pm \pm} \gamma}^{ \pm \pm}+\Gamma_{U^{ \pm \pm} h^{0}}^{ \pm \pm}+\Gamma_{U^{ \pm \pm} H_{1}^{0}}^{ \pm \pm}+\Gamma_{U^{ \pm \pm} H_{2}^{0}}^{ \pm \pm}+\Gamma_{U^{ \pm \pm}}^{ \pm \pm} H_{3}^{0} \\
& +\Gamma_{U^{ \pm \pm} Z}^{ \pm \pm}+\Gamma_{U^{ \pm \pm} Z^{\prime}}^{ \pm \pm}+\Gamma_{V^{ \pm} H_{1}^{ \pm}}^{ \pm \pm}
\end{aligned}
$$

with $\Gamma_{X Y}^{ \pm \pm}=\Gamma\left(H^{ \pm \pm} \rightarrow X Y\right)$ (see Appendix A for the partial widths).

The contribution for the interference of the $H_{1}^{0}$ and $H_{2}^{0}$ is given by

$$
\begin{aligned}
\frac{d \hat{\sigma}_{H_{1}^{0}-H_{2}^{0}}}{d \Omega}= & \sum_{q_{u}, q_{d}} \frac{\alpha_{s}^{4} \Lambda_{1} \Lambda_{2} \Lambda_{k_{1}} \Lambda_{k_{2}} \zeta^{(2)}(\hat{s}) \delta_{a b}}{8 \pi} \\
& \times\left\{\frac{\varepsilon_{k_{1}}^{\mu(a)} \varepsilon_{k_{2}}^{v(b)} k_{1}^{\alpha} k_{2}^{\beta}}{\hat{s}^{2}} m_{q}^{2} I_{q}+i \frac{\varepsilon_{k_{1}}^{a} \varepsilon_{k_{2}}^{b} \delta_{a b}}{2} \lambda\left[2+(4 \lambda-1) I_{q}\right]\right\} \\
& \times\left\{\frac{\varepsilon_{k_{1}}^{\mu(a)} \varepsilon_{k_{2}}^{v(b)} k_{1}^{\alpha} k_{2}^{\beta} \varepsilon_{\alpha \mu \nu \beta}}{\hat{s}^{2}}\left(\frac{v_{\eta}}{v_{\rho}} m_{u}^{2} I_{q_{u}}-\frac{v_{\rho}}{v_{\eta}} m_{d}^{2} I_{q_{d}}\right)\right. \\
& \left.+i \frac{\varepsilon_{k_{1}}^{a} \varepsilon_{k_{2}}^{b}}{2}\left[\frac{v_{\eta}}{v_{\rho}} \lambda_{U}\left[2+\left(4 \lambda_{U}-1\right) I_{q_{u}}\right]-\frac{v_{\rho}}{v_{\eta}} \lambda_{D}\left[2+\left(4 \lambda_{D}-1\right) I_{q_{d}}\right]\right]\right\},
\end{aligned}
$$

where $\varepsilon^{\mu, \nu}$ are the polarizations, $k_{1,2}$ are the gluon momentum vectors, $m_{q_{u}}$ are the masses of the $u, c, t$ quarks ( $5 \mathrm{MeV}, 1.5 \mathrm{GeV}$ and $175 \mathrm{GeV}$ respectively), $m_{q_{d}}$ are the masses of the $d, s$, $b$ quarks (9 MeV, $150 \mathrm{MeV}$ and $5 \mathrm{GeV}$ respectively) [14], $\lambda$ is referred to all quarks and $\lambda_{U}$ for the quark $u, c, t$ and $\lambda_{D}$ for $d, s, b$ respectively and $\varepsilon_{\alpha \mu \nu \beta}$ is the antisymmetric tensor.

The contribution for the interference of $H_{1}^{0}$ and $H_{3}^{0}$ gives

$$
\begin{aligned}
\frac{d \hat{\sigma}_{H_{1}^{0}-H_{3}^{0}}}{d \Omega}= & \sum_{q} i \frac{\alpha_{s}^{4} \Lambda_{1} \Lambda_{3} \Lambda_{q_{1}} \Lambda_{q_{3}} \zeta^{(1)}(\hat{s}) \zeta^{(3)} \delta_{a^{\prime} b^{\prime}} \varepsilon_{q_{1}}^{a^{\prime}} \varepsilon_{q_{2}}^{b^{\prime}} \delta_{a b}}{256 \pi^{2}} \lambda\left[2+(4 \lambda-1) I_{q}\right] \\
& \times\left\{i \frac{\varepsilon_{q_{1}}^{a} \varepsilon_{q_{2}}^{b}}{2} \lambda\left[2+(4 \lambda-1) I_{q}\right]-\frac{\varepsilon_{q_{1}}^{\mu(a)} \varepsilon_{q_{2}}^{(b)} \varepsilon_{\alpha \mu \nu \beta}}{\hat{s}^{2}}\right\},
\end{aligned}
$$

and finally we have the following for the $H_{2}^{0}$ and $H_{3}^{0}$

$$
\begin{aligned}
\frac{d \hat{\sigma}_{H_{2}^{0}-H_{3}^{0}}}{d \Omega}= & \sum_{q} i \frac{\alpha_{s}^{4} \Lambda_{2} \Lambda_{3} \Lambda_{q_{2}} \Lambda_{q_{3}} \zeta^{(2)}(\hat{s}) \zeta^{(3)}(\hat{s}) \delta_{a^{\prime} b^{\prime}} \varepsilon_{q_{1}}^{a^{\prime}} \varepsilon_{q_{2}}^{b^{\prime}} \delta_{a b}}{256 \pi^{2}} \lambda\left[2+4(4 \lambda-1) I_{q}\right] \\
& \times\left\{\varepsilon_{q_{1}}^{\mu(a)} \varepsilon_{q_{2}}^{\nu(b)} \varepsilon_{\alpha \mu \nu \beta}\left(\frac{v_{\eta}}{v_{\rho}} m_{u}^{2} I_{q_{u}}-\frac{v_{\rho}}{v_{\eta}} m_{d}^{2} I_{q_{d}}\right)\right. \\
& \left.+i \frac{\varepsilon_{q_{1}}^{a} \varepsilon_{q_{2}}^{b}}{2}\left[\frac{v_{\eta}}{v_{\rho}} \lambda_{u}\left[2+\left(4 \lambda_{u}-1\right) I_{q_{u}}\right]-\frac{v_{\rho}}{v_{\eta}} \lambda_{d}\left[2+\left(4 \lambda_{d}-1\right) I_{q_{u}}\right]\right]\right\} .
\end{aligned}
$$


The loop integrals involved in the evaluation of the elementary cross section can be expressed in terms of the function $I_{q}\left(\lambda_{q}\right) \equiv I_{q}$ which is defined through

$$
\begin{aligned}
I_{q} & =\int_{0}^{1} \frac{d x}{x} \ln \left[1-\frac{(1-x) x}{\lambda_{q}}\right] \\
& =\frac{1}{2} \begin{cases}-4 \arcsin ^{2}\left[1 /\left(2 \sqrt{\lambda_{q}}\right)\right], & \lambda_{q}>1 / 4, \\
{\left[\ln \left(r_{+q} / r_{-q}\right)+2 i \pi\right] \ln \left(r_{+q} / r_{-q}\right)-\pi^{2},} & \lambda_{q}<1 / 4 .\end{cases}
\end{aligned}
$$

Here, $q$ stands for the quarks running in the loop (Fig. 1(b)).

The total cross section $(\sigma)$ for the process $p p \rightarrow g g \rightarrow H^{--} H^{++}$is related to the total cross section $(\hat{\sigma})$ through the subprocess $g g \rightarrow H^{--} H^{++}$, i.e.,

$$
\sigma=\int_{\tau_{\min }}^{1} \int_{\ln \sqrt{\tau_{\min }}}^{-\ln \sqrt{\tau_{\min }}} d \tau d y G\left(\sqrt{\tau} e^{y}, Q^{2}\right) G\left(\sqrt{\tau} e^{-y}, Q^{2}\right) \hat{\sigma}(\tau, \hat{s}),
$$

where $G\left(x, Q^{2}\right)$ is the gluon structure function, for which the factorization scale is taken equal to the center of mass energy of the subprocess and used in our numerical calculations.

\section{Results and conclusions}

In this work we have calculated the pair production of doubly charged Higgs by computing the contributions due the Drell-Yan and quark loop processes. In Section 3 we have given the analytical expressions that allow the numerical evaluations of these contributions and it was showed that the dominant contribution come from the well-known Drell-Yan process. We have presented the cross section for the process $p p \rightarrow H^{--} H^{++}$involving the Drell-Yan mechanism and the gluon-gluon fusion, to produce such Higgs bosons at the LHC (14 TeV).

Taking into account that the masses of the gauge bosons, Higgs and some other parameters must satisfy the limits imposed by the model (see Section 2), besides the approximations in the calculations of masses (Appendix B) and eigenstates (Section 2) given in Ref. [12], we have considered two possibilities: $f \simeq 0$ and $f=-99.63 \mathrm{GeV}$ (see Table 1). For both possibilities we have assumed the values $v_{\eta}=195 \mathrm{GeV}, v_{\chi}=1300 \mathrm{GeV}, \lambda_{1}=-1.2,-\lambda_{2}=-\lambda_{3}=\lambda_{6}=$ $-\lambda_{8}=1, \lambda_{4}=+2.98, \lambda_{5}=-1.56, \lambda_{7}=-3$, but for $\lambda_{9}$ we have used $\lambda_{9}=-1$ when $f \approx 0$ and $\lambda_{9}=-1.9$ when $f=-99.63 \mathrm{GeV}$.

The masses of the exotic bosons in Table 1 are in accordance with the estimated values of $\mathrm{CDF}$ and $\mathrm{D} \varnothing$ experiments, which probe their masses in the range from $500 \mathrm{GeV}$ to $800 \mathrm{GeV}$ [17], while the reach of the $\mathrm{LHC}$ will be in the range $1 \mathrm{TeV}<m_{Z^{\prime}} \leqslant 5 \mathrm{TeV}$ [18].

In Fig. 2 we show the cross section for the process $p p \rightarrow H^{++} H^{--}$for $f \simeq 0 \mathrm{GeV}$. Considering that the expected integrated luminosity for the LHC will be of order of $3 \times 10^{5} \mathrm{pb}^{-1} / \mathrm{yr}$

Table 1

Approximated values of the masses (see Appendix B) used in this work. All the values in this table are given in GeV

\begin{tabular}{lccccccccccccccc}
\hline$f$ & $m_{E}$ & $m_{M}$ & $m_{T}$ & $m_{H_{1}^{0}}$ & $m_{H_{2}^{0}}$ & $m_{H_{3}^{0}}$ & $m_{h^{0}}$ & $m_{H_{1}^{ \pm}}$ & $m_{H_{2}^{ \pm}}$ & $m_{V}$ & $m_{U}$ & $m_{Z^{\prime}}$ & $m_{J_{1}}$ & $m_{J_{2}}$ & $m_{J_{3}}$ \\
\hline$\approx 0$ & 194 & 1138 & 2600 & 874 & 1322 & 2600 & 0 & 426 & 1315 & 603 & 601 & 2220 & 1300 & 1833 & 1833 \\
-99.63 & & & & & & & 520 & 218 & 1295 & & & & & & \\
\hline
\end{tabular}




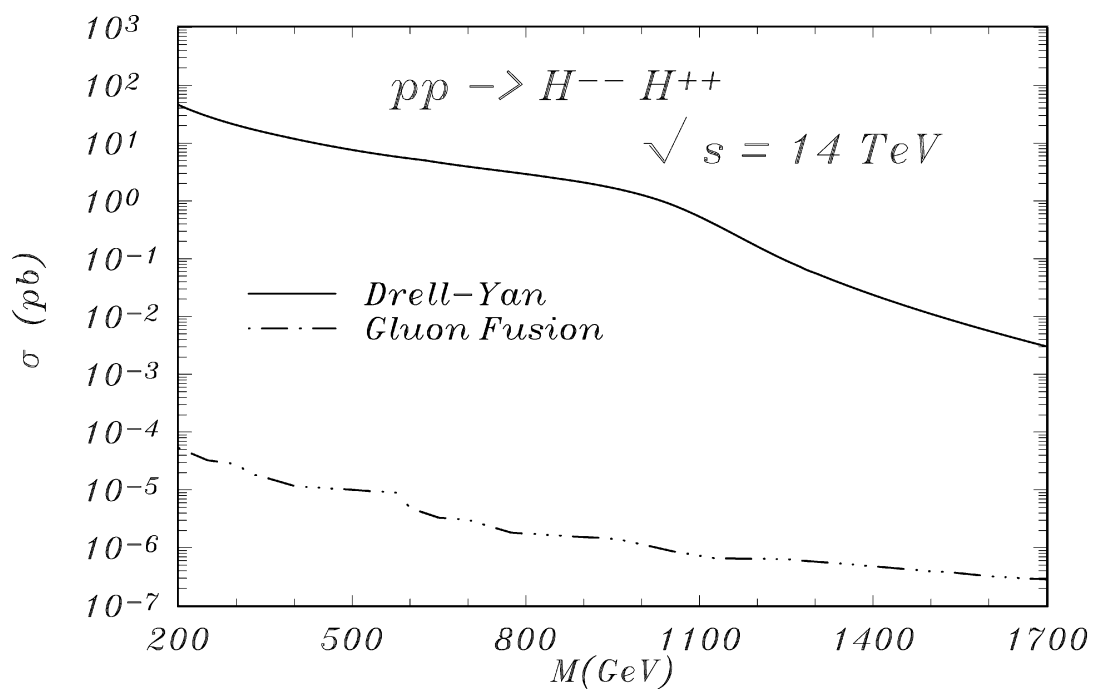

Fig. 2. Total cross section for the process $p p \rightarrow H^{--} H^{++}$as a function of $m_{H^{ \pm \pm}}$for $f=0 \mathrm{GeV}$ at $\sqrt{s}=14000 \mathrm{GeV}$ for Drell-Yan (solid line) and gluon-gluon fusion (dot-dash line).

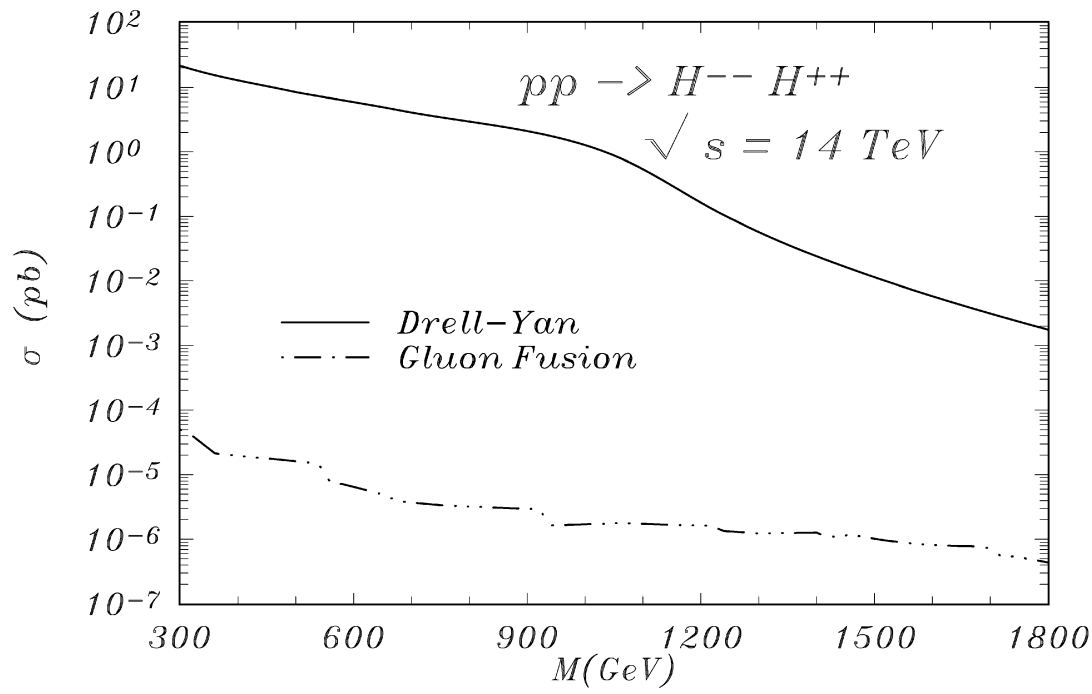

Fig. 3. Total cross section for the process $p p \rightarrow H^{--} H^{++}$as a function of $m_{H^{ \pm \pm}}$for $f=-99.63 \mathrm{GeV}$ at $\sqrt{s}=14000 \mathrm{GeV}$ for Drell-Yan (solid line) and gluon-gluon fusion (dot-dash line).

then the statistics give a total of $\simeq 2 \times 10^{4}$ events per year for Drell-Yan and $\simeq 0.2$ events per year for gluon-gluon fusion, for $m_{H^{ \pm}}=1309 \mathrm{GeV}$.

In Fig. 3, we show the results for the same process when $f=-99.67 \mathrm{GeV}$. This value was calculated considering the exotic boson masses in the range from 500 to $800 \mathrm{GeV}$ and $v_{\eta}$ having a minimum value of $194.5 \mathrm{GeV}$, which assure the values of the $\lambda_{i}$ between -3 to +3 , so we obtain the mass of the doubly charged Higgs, i.e., $m_{H^{++}}=1780 \mathrm{GeV}$. Considering the same integrated luminosity as above this gives a total of 585 events per year for Drell-Yan and 0.13 events per 
Table 2

Branching ratios for the $H_{3}^{0}$ decay with $m_{H_{3}^{0}}=2600 \mathrm{GeV}$. The notation used in this table is $B R_{X Y}^{0}=B R\left(H_{3}^{0} \rightarrow X Y\right)$

\begin{tabular}{lllllll}
\hline$f(\mathrm{GeV})$ & $10^{-5} \times B R_{H_{1}^{0} H_{2}^{0}}^{0}$ & $10^{-8} \times B R_{H_{1}^{+} H_{1}^{-}}^{0}$ & $B R_{2}^{0} H_{2}^{+}$ & $B R_{1}^{0} H_{2}^{-}$ & $B R_{E^{+} E^{-}}^{0}$ & $B R_{M^{+} M^{-}}^{0}$ \\
\hline$\approx 0$ & 3.35 & 2 & no & 0.9999 & $2 \times 10^{-7}$ & $2 \times 10^{-6}$ \\
-99.63 & 3.14 & 4 & $4 \times 10^{-7}$ & & & \\
\hline
\end{tabular}

Table 3

Branching ratio for the $H^{ \pm \pm}$decay with $m_{H^{ \pm \pm}}=1309 \mathrm{GeV}$. Here $B R_{X Y}^{ \pm \pm}=10^{-3} \times B R\left(H^{ \pm \pm} \rightarrow X Y\right)$

\begin{tabular}{llllllc}
\hline$f(\mathrm{GeV})$ & $B R_{J_{1} q}^{ \pm \pm}$ & $B R_{\ell^{-} E^{-}}^{--}$ & $B R_{\ell^{-} M^{-}}^{--}$ & $B R_{\ell^{+} E^{+}}^{++}$ & $B R_{\ell^{+}}^{++} M^{+}$ & $B R_{U^{ \pm \pm}}^{ \pm \pm}$ \\
\hline$\approx 0$ & 0.001 & 0.08 & 0.005 & 3 & 6 & 29 \\
-99.63 & 2 & 0.001 & 0.004 & 0.4 & 4 & 2 \\
$f(\mathrm{GeV})$ & $B R_{W^{ \pm}}^{ \pm} H_{2}^{ \pm}$ & $B R_{V^{ \pm}}^{ \pm \pm} H_{1}^{ \pm}$ & $B R_{H_{1}^{ \pm}}^{ \pm \pm}$ & $B R_{U^{ \pm \pm} H_{1}^{0}}^{ \pm \pm}$ & $B R_{U^{ \pm \pm} Z}^{ \pm \pm}$ & $B R_{U^{ \pm \pm} h^{0}}^{ \pm}$ \\
\hline$\approx 0$ & no & 19 & no & no & 444 & 2 \\
-99.63 & 0.09 & 13 & 329 & 6 & 146 & 0.5 \\
\hline
\end{tabular}

year for gluon-gluon fusion. We present in Table 2 the branching ratios for $H_{3}^{0} \rightarrow$ all with $f \simeq 0$ and $f=-99.63 \mathrm{GeV}$ and we can observe that, due to the coupling constant, the largest width corresponds to $H_{3}^{0} \rightarrow H_{1}^{+} H_{2}^{-}$decay. In Table 3 we present the branching ratios for $H^{ \pm \pm} \rightarrow$ all. From Table 3 it can also be noticed that, as the branching ratio (BR) for $H^{ \pm \pm} \rightarrow H_{1}^{ \pm} H_{2}^{ \pm}$, $H^{ \pm \pm} \rightarrow U^{ \pm \pm} Z$ and $H^{ \pm \pm} \rightarrow U^{ \pm \pm} \gamma$ are large, these channels could lead to some interesting signal.

We emphasize that the window for varying the free parameters is small because of the constraints imposed on the model. In summary, the analysis of the values in Tables 1-3 show that, although a large number of doubly charged Higgs can be produced by the Drell-Yan mechanism, the decays of these particles into ordinary quarks and leptons do not lead to a good signature for its detection even for energies which the LHC can reach.

\section{Appendix A. Partial widths}

In this appendix we present the partial widths for Higgs decays from Eqs. (23) and (24). We define

$$
R(a, b ; x)=\frac{1}{16 \pi x^{3 / 2}} \sqrt{\left[x-\left(m_{a}+m_{b}\right)^{2}\right]\left[x-\left(m_{a}-m_{b}\right)^{2}\right]}
$$

and we obtain the partial widths for $H_{3}^{0}$, with $\sqrt{s}=m_{H_{3}^{0}}$ as

$$
\begin{aligned}
& \Gamma_{\bar{J}_{i} J_{i}}^{0}=3 R\left(J_{i}, J_{i} ; s\right)\left(\frac{m_{J_{i}}}{v_{\chi}}\right)^{2}\left(m_{H_{3}^{0}}^{2}-2 m_{J_{i}}^{2}\right), \\
& \Gamma_{P^{+} P^{-}}^{0}=R\left(P^{+}, P^{-} ; s\right)\left(\frac{m_{P}}{v_{\chi}}\right)^{2}\left(m_{H_{3}^{0}}^{2}-2 m_{P}\right), \\
& \Gamma_{H_{1}^{0} H_{2}^{0}}^{0}=R\left(H_{1}^{0}, H_{2}^{0} ; s\right)\left[4\left(\lambda_{5}-\lambda_{6}\right) c_{\omega} v_{\rho} v_{\chi}+f\left(c_{\omega} v_{\eta}-s_{\omega} v_{\rho}\right)\right]^{2}, \\
& \Gamma_{H_{1}^{-} H_{1}^{+}}^{0}=R\left(H_{1}^{+}, H_{1}^{+} ; s\right)\left[2 v_{\chi}\left(\lambda_{5} s_{\omega} v_{\rho}+\lambda_{6} c_{\omega} v_{\eta}\right)+f c_{\omega} v_{\rho}\right]^{2},
\end{aligned}
$$




$$
\begin{aligned}
& \Gamma_{H_{2}^{-} H_{2}^{+}}^{0}=R\left(H_{2}^{+}, H_{2}^{+} ; s\right)\left[-2 v_{\chi}\left(\lambda_{5} c_{\omega} v_{\eta}+\lambda_{6} s_{\omega} v_{\rho}\right)+f s_{\omega} v_{\eta}\right]^{2}, \\
& \Gamma_{H_{1}^{-} H_{2}^{+}}^{0}=R\left(H_{1}^{+}, H_{2}^{+} ; s\right)\left[\left(\lambda_{6}-\lambda_{5}\right) c_{\omega} v_{\rho} v_{\chi}+f\left(s_{\omega} v_{\rho}-c_{\omega} v_{\eta}\right)\right] \\
& \Gamma_{U^{--} U^{++}}^{0}=\frac{g^{4} v_{\chi}}{2} R\left(U^{ \pm \pm}, U^{ \pm \pm} ; s\right)\left\{3-\left(\frac{m_{H_{3}^{0}}}{m_{U^{++}}}\right)^{2}\left[1-\left(\frac{m_{H_{3}^{0}}}{2 m_{U^{++}}}\right)^{2}\right]\right\} \\
& \Gamma_{H^{--} H^{++}}^{0}=4 v_{\chi} R\left(H^{ \pm \pm}, H^{ \pm \pm} ; s\right)\left[\left(\lambda_{6}+\lambda_{9}\right) s_{\varphi}^{2}+\left(2 \lambda_{3}+\lambda_{9}\right) c_{\varphi}^{2}\right]
\end{aligned}
$$

where $\Gamma_{X Y}^{0} \equiv \Gamma\left(H_{3}^{0} \rightarrow X Y\right)$. Finally we present the partial widths for the $H^{--}$decays, with $\sqrt{s}=m_{H^{++}}$,

$$
\begin{aligned}
& \Gamma_{\bar{J}_{1} q_{d, s, b}}^{++}=3 R\left(J_{1}, q ; s\right)\left(\frac{m_{q} s_{\varphi}}{v_{\eta}}\right)^{2}\left(m_{H^{++}}^{2}-m_{J_{1}}^{2}-m_{d, s, b}^{2}\right), \\
& \Gamma_{\bar{q}_{u, c, t} J_{2,3}}^{++}=3 R\left(J_{2,3}, q ; s\right)\left(\frac{m_{J_{2,3} c_{\varphi}}}{v_{\chi}}\right)^{2}\left(m_{H^{++}}^{2}-m_{J_{2,3}}^{2}-m_{u, c, t}^{2}\right), \\
& \Gamma_{\bar{J}_{2,3} q_{u, c, t}}^{++}=3 R\left(J_{2,3}, q ; s\right)\left(\frac{m_{q_{u, c, t}}^{2} s_{\varphi}}{v_{\eta}}\right)^{2}\left(m_{H^{++}}^{2}-m_{J_{2,3}}^{2}-m_{u, c, t}^{2}\right) \text {, } \\
& \Gamma_{e^{-} P^{-}}^{++}=\frac{R(e, P ; s)}{4}\left(\frac{m_{e} s_{\varphi}}{v_{\eta}}\right)^{2}\left(m_{H^{++}}^{2}-m_{e}^{2}-m_{P}^{2}\right), \\
& \Gamma_{e^{+} P^{+}}^{++}=\frac{R(e, P ; s)}{4}\left(\frac{m_{P} c_{\varphi}}{v_{\chi}}\right)^{2}\left(m_{H^{++}}^{2}-m_{e}^{2}-m_{P}^{2}\right), \\
& \Gamma_{W^{-} H_{2}^{-}}^{++}=\frac{R\left(W, H_{2}^{-} ; s\right)}{32}\left(\frac{e c_{\varphi} c_{\phi}}{\sin \theta_{W} m_{W}}\right)^{2} \\
& \times\left\{\left(m_{H^{++}}^{2}-m_{W}^{2}\right)^{2}+m_{H_{2}^{+}}^{2}\left[m_{H_{2}^{+}}^{2}-\left(m_{H^{++}}^{2}+m_{W}^{2}\right)\right]\right\}, \\
& \Gamma_{H_{1}^{-} H_{2}^{-}}^{++}=R\left(H_{2}^{-}, H_{2}^{-} ; s\right)\left\{\left[\left(\lambda_{7}+\lambda_{9}\right) s_{\omega}^{2}+\left(\lambda_{7}+\lambda_{8}\right) c_{\omega}^{2}\right] s_{\phi} s_{\varphi}\right. \\
& \left.+\frac{\left(\lambda_{8}-\lambda_{9}\right) c_{\omega} c_{\phi} v_{\rho}+f s_{\omega} s_{\phi}}{\sqrt{v_{\eta}^{2}+v_{\chi}^{2}}}\right\} \\
& \Gamma_{U^{--} \gamma}^{++}=\frac{3}{4 \pi m_{H^{++}}}\left(\frac{e^{2} c_{\varphi} v_{\chi}}{\sin ^{2} \theta_{W}}\right)^{2}\left[1-\left(\frac{m_{U^{++}}}{m_{H^{++}}}\right)^{2}\right] \text {, } \\
& \Gamma_{U^{--} H_{1}^{0}}^{++}=\frac{R\left(U^{--}, H_{1}^{0} ; s\right)}{32}\left(\frac{e c_{\varphi} v_{\chi}}{m_{U^{++}} v_{W} \sin \theta_{W}}\right)^{2} \\
& \times\left\{\left(m_{H^{++}}^{2}-m_{U^{++}}^{2}\right)^{2}+m_{H_{1}^{0}}^{2}\left[m_{H_{1}^{0}}^{2}-2\left(m_{H^{++}}^{2}+m_{U^{--}}^{2}\right)\right]\right\}, \\
& \Gamma_{U^{--} H_{2}^{0}}^{++}=\frac{R\left(U^{--}, H_{2}^{0} ; s\right)}{32}\left(\frac{e c_{\phi} v_{\rho}}{m_{U^{++}} v_{W} \sin \theta_{W}}\right)^{2} \\
& \times\left\{\left(m_{H^{++}}^{2}-m_{U^{--}}^{2}\right)^{2}+m_{H_{2}^{0}}^{2}\left[m_{H_{1}^{0}}^{2}-2\left(m_{H^{++}}^{2}+m_{U^{++}}^{2}\right)\right]\right\},
\end{aligned}
$$




$$
\begin{aligned}
\Gamma_{U^{--} H_{3}^{0}}^{++} & \frac{R\left(U^{--}, H_{3}^{0} ; s\right)}{32}\left(\frac{e c_{\varphi}}{m_{U^{++}} \sin ^{2} \theta_{W}}\right)^{2} \\
& \times\left\{\left(m_{H^{++}}^{2}-m_{U^{++}}^{2}\right)^{2}+m_{H^{0}}^{2}\left[m_{H_{3}^{0}}^{2}-2\left(m_{H^{++}}^{2}+m_{U^{++}}^{2}\right)\right]\right\} \\
\Gamma_{U^{--} Z}^{++}= & R\left(U^{--}, Z ; s\right)\left(\frac{e^{2} c_{\varphi} v_{\chi}}{\cos \theta}\right)^{2} \\
& \times\left[5+\frac{\left(m_{H^{++}}^{2}-m_{Z}^{2}\right)^{2}+m_{U^{++}}^{2}\left(m_{U^{++}}^{2}-2 m_{H^{++}}^{2}\right)}{2 m_{U^{++}}^{2} m_{Z}^{2}}\right] \\
\Gamma_{U^{--} Z^{\prime}}^{++} & \frac{R\left(U^{--}, Z^{\prime} ; s\right)}{12\left(\sin ^{2} \theta_{W}-1\right)\left(4 \sin ^{2} \theta_{W}-1\right)}\left[\frac{e v_{\eta} v_{\chi}\left(10 \sin ^{2} \theta_{W}-1\right)}{\sin ^{2} \theta_{W}}\right]^{2} \\
& \times\left[5+\frac{\left(m_{H^{++}}^{2}-m_{Z^{\prime}}^{2}\right)^{2}+m_{U^{++}}^{2}\left(m_{U^{++}}^{2}-2 m_{H^{++}}^{2}\right)}{2 m_{U^{++}}^{2} m_{Z^{\prime}}^{2}}\right] \\
& \times\left\{\left(m_{H^{++}}^{2}-m_{V}^{2}\right)^{2}+m_{H_{1}^{0}}^{2}\left[m_{H_{1}^{+}}^{2}-2\left(m_{H^{++}}^{2}+m_{V}^{2}\right)\right]\right\}
\end{aligned}
$$

Here, $\Gamma_{X Y}^{++} \equiv \Gamma\left(H^{++} \rightarrow X Y\right)$.

\section{Appendix B. 3-3-1 particle masses}

In this appendix we present the expressions of gauge, Higgs boson and fermion masses predicted in 3-3-1 energy scale in terms of the VEVs and the parameters of the potential.

$$
\begin{aligned}
& m_{H_{1}^{0}}^{2} \approx 4 \frac{\lambda_{2} v_{\rho}^{4}-2 \lambda_{1} v_{\eta}^{4}}{v_{\eta}^{2}-v_{\rho}^{2}}, \quad m_{H_{2}^{0}}^{2} \approx \frac{v_{W}^{2}}{2 v_{\eta} v_{\rho}^{2}} v_{\chi}^{2}, \quad m_{H_{3}^{0}}^{2} \approx-4 \lambda_{3} v_{\chi}^{2}, \\
& m_{h}^{2}=-\frac{f v_{\chi}}{v_{\eta} v_{\rho}}\left[v_{W}^{2}+\left(\frac{v_{\eta} v_{\rho}}{v_{\chi}}\right)^{2}\right], \quad m_{H_{1}^{ \pm}}^{2}=\frac{v_{W}^{2}}{2 v_{\eta} v_{\rho}}\left(f v_{\chi}-2 \lambda_{7} v_{\eta} v_{\rho}\right), \\
& m_{H_{2}^{ \pm}}=\frac{v_{\eta}^{2}+v_{\chi}^{2}}{2 v_{\eta} v_{\chi}}\left(f v_{\rho}-2 \lambda_{8} v_{\eta} v_{\chi}\right), \quad m_{H^{ \pm \pm}}^{2}=\frac{v_{\rho}^{2}+v_{\chi}^{2}}{2 v_{\rho} v_{\chi}}\left(f v_{\eta}-2 \lambda_{9} v_{\rho} v_{\chi}\right), \\
& m_{W}^{2}=\frac{1}{2}\left(\frac{e v_{W}}{s_{W}}\right)^{2}, \quad m_{V}^{2}=\left(\frac{e}{s_{W}}\right)^{2} \frac{v_{\eta}^{2}+v_{\chi}^{2}}{2}, \quad m_{U}^{2}=\left(\frac{e}{s_{W}}\right)^{2} \frac{v_{\rho}^{2}+v_{\chi}^{2}}{2}, \\
& m_{Z}^{2} \approx\left(\frac{e v_{\eta}}{s_{W}}\right)^{2} \frac{1}{2\left(1-s_{W}\right)}, \quad m_{Z^{\prime}}^{2} \approx\left(\frac{e v_{\chi}}{s_{W}}\right)^{2} \frac{2\left(1-s_{W}^{2}\right)}{3\left(1-4 s_{W}^{2}\right)} .
\end{aligned}
$$

In the calculations of Ref. [12] the following conditions in imposed:

$$
\lambda_{4} \approx 2 \frac{\lambda_{2} v_{\rho}^{2}-\lambda_{1} v_{\eta}^{2}}{v_{\eta}^{2}-v_{\rho}^{2}}, \quad \lambda_{5} v_{\eta}^{2}+2 \lambda_{6} v_{\rho}^{2} \approx-\frac{v_{\eta} v_{\rho}}{2} .
$$

From the Lagrangian (9a) we can see that $m_{e}, m_{\mu}, m_{\tau} \propto v_{\rho}$ and $m_{E}, m_{M}, m_{T} \propto v_{\chi}$. Concerning the ordinary quarks the masses can be obtained from the Lagrangian (9b) taking into account 
$m_{u} \ll m_{c} \ll m_{t}$ and $m_{d} \ll m_{s} \ll m_{b}$. Therefore, we have

$$
\begin{aligned}
& m_{u}=\frac{\mathcal{G}_{1}^{u} v_{\eta} v_{\rho}}{\mathcal{G}_{2}^{u} v_{\rho}+\mathcal{G}_{3}^{u} v_{\eta}}, \quad m_{c}=\frac{\mathcal{G}_{1}^{c} v_{\rho}+\mathcal{G}_{2}^{c} v_{\eta}}{\mathcal{G}_{3}^{c} v_{\eta}+\mathcal{G}_{4}^{c} v_{\rho}} v_{\rho}, \quad m_{t}=\mathcal{G}_{1}^{t} v_{\eta}+\mathcal{G}_{2}^{t} v_{\rho}, \\
& m_{d}=\frac{\mathcal{G}_{1}^{d} v_{\rho} v_{\eta}}{\mathcal{G}_{2}^{d} v_{\eta}+\mathcal{G}_{3}^{d} v_{\rho}}, \quad m_{s}=\frac{\mathcal{G}_{1}^{s} v_{\eta}+\mathcal{G}_{2}^{s} v_{\rho}}{\mathcal{G}_{3}^{s} v_{\rho}+\mathcal{G}_{4}^{s} v_{\eta}} v_{\eta}, \quad m_{b}=\mathcal{G}_{1}^{b} v_{\rho}+\mathcal{G}_{2}^{b} v_{\eta},
\end{aligned}
$$

and from the Lagrangian (9c) the heavy quark masses are $m_{J_{1}}, m_{J_{2}}, m_{J_{3}} \propto v_{\chi}$. In Eqs. (B.3) the parameters $\mathcal{G}_{\beta}^{q}, q=u, c, t, d, s, b$ and $\beta=1,2,3,4$ are functions of the Yukawa couplings.

\section{References}

[1] J. Wess, B. Zumino, Nucl. Phys. B 70 (1974) 39;

J. Wess, B. Zumino, Phys. Lett. B 49 (1974) 52;

J. Iliopoulos, B. Zumino, Nucl. Phys. B 76 (1974) 310;

S. Ferrara, J. Iliopoulos, B. Zumino, Nucl. Phys. B 77 (1974) 413;

E. Witten, Nucl. Phys. B 188 (1981) 513.

[2] N. Arkani-Hamed, et al., JHEP 0208 (2002) 021;

M. Scmaltz, Nucl. Phys. B (Proc. Suppl.) 117 (2003) 40.

[3] J.C. Pati, A. Salam, Phys. Rev. D 10 (1974) 275;

R.N. Mohapatra, J.C. Pati, Phys. Rev. D 11 (1975) 566;

G. Senjanovic, R.N. Mohapatra, Phys. Rev. D 12 (1975) 1502;

T. Rizzo, Phys. Rev. D 25 (1982) 1355.

[4] H. Georgi, M. Machacek, Nucl. Phys. B 262 (1985) 463;

J.F. Gunion, R. Vega, J. Wudka, Phys. Rev D 42 (1990) 1673;

J.F. Gunion, C. Loomis, K.T. Pitts, hep-ph/9610237.

[5] F. Pisano, V. Pleitez, Phys. Rev. D 46 (1992) 410;

P.H. Frampton, Phys. Rev. Lett. 69 (1992) 2889;

R. Foot, O.F. Hernandez, F. Pisano, V. Pleitez, Phys. Rev. Lett. 47 (1993) 4158.

[6] V. Pleitez, M.D. Tonasse, Phys. Rev. D 48 (1993) 2353.

[7] A.G. Dias, R. Martinez, V. Pleitez, Eur. Phys. J. C 39 (2005) 101;

P. Jain, S.D. Joglekar, Phys. Lett. B 407 (1997) 151.

[8] N.V. Cortez, M.D. Tonasse, Phys. Rev. D 72 (2005) 073005;

J.C. Montero, C.A. de S. Pires, V. Pleitez, Phys. Rev. D 65 (2002) 095001.

[9] For some recently papers see A.G. Akeroyd, M. Aoki, Phys. Rev. D 72 (2005) 035011;

CDF Collaboration, D. Acosta, et al., Phys. Rev. Lett. 95 (2005) 0151801;

CDF Collaboration, D. Acosta, et al., Phys. Rev. Lett. 93 (2004) 221802;

DØ Collaboration, V.M. Abazov, et al., Phys. Rev. Lett. 93 (2004) 141801;

L3 Collaboration, P. Achard, et al., Phys. Lett. B 576 (2003) 18;

OPAL Collaboration, G. Abbiendi, et al., Phys. Lett. B 577 (2003) 93.

[10] J.C. Montero, F. Pisano, V. Pleitez, Phys. Rev. D 47 (1993) 2918;

H.N. Long, Phys. Rev. D 59 (1996) 437;

R. Foot, H.N. Long, T.A. Tran, Phys. Rev. D 50 (1994) 34.

[11] V. Pleitez, M.D. Tonasse, Phys. Rev. D 48 (1993) 5274.

[12] M.D. Tonasse, Phys. Lett. B 381 (1996) 191.

[13] P.V. Dong, H.L. Long, D.T. Nhung, Phys. Rev. D 73 (2006) 035004;

R.A. Diaz, R. Martinez, F. Ochoa, Phys. Rev. D 69 (2006) 095009, and references cited therein.

[14] Particle Data Group, S. Eidelman, et al., Phys. Lett. B 592 (2004) 1.

[15] J.E. Cieza Montalvo, M.D. Tonasse, Nucl. Phys. B 623 (2002) 325.

[16] J.E. Cieza Montalvo, M.D. Tonasse, Phys. Rev. D 71 (2005) 095015.

[17] M. Carena, A. Daleo, B.A. Dobrescu, T.M.P. Tait, Phys. Rev. D 70 (2004) 093009;

DØ Collaboration, V.M. Abazov, et al., Phys. Rev. Lett. 87 (2001) 061802;

CDF Collaboration, T. Pratt, Talk at the SUSY 2004 Conference, June 2004.

[18] A. Freitas, Phys. Rev. D 70 (2004) 015008. 